\title{
DIABETES MELLITUS, OBESIDADE E MAUS HÁBITOS DE VIDA: SUA RELAÇÃO COM A COVID-19
}

\author{
DIABETES MELLITUS, OBESITY AND BAD LIFESTYLE HABITS: \\ THEIR RELATIONSHIP WITH COVID-19
}

Amanda Rainha Monteiro', Ludmila Alves dos Santos Barros ${ }^{1}$, Pedro Cardoso Siqueira Albernaz', Victor Barcellos de Azeredo Vieira', Victoria Dias de Miranda Paula Barroso', Marta Cristina da Cunha-Rodrigues ${ }^{2}$

1. Acadêmicos do curso de Medicina.

2- Doutora em Fisiopatologia Clínica e Experimental. Docente dos componentes curriculares de Fisiologia e

Farmacologia.

Faculdade de Medicina de Campos - FMC

Av. Alberto Torres, 217 - Centro, Campos dos Goytacazes - RJ, 28035-581

Autora correspondente: Marta C. da Cunha-Rodrigues - Contato: (21) 984369757, marta.rodrigues@fmc.br Faculdade de Medicina de Campos - FMC - Av. Alberto Torres, 217 - Centro, Campos dos Goytacazes - RJ, 28035581

\section{RESUMO}

No ano de 2019 foi descoberto o vírus que viria a se chamar de SARS-CoV-2 e que provocou uma crise sanitária global. A pandemia de 2020, causada pela doença nomeada de Coronavírus disease 2019 ou COVID-19 alastrou-se rapidamente ao redor do mundo, impactando a população global e, especialmente, alguns grupos de risco, como pacientes com doenças cardiovasculares, obesos e diabéticos, por exemplo. Este estudo possui o objetivo de analisar e relatar as relações existentes entre a COVID-19 e os pacientes que possuem distúrbios metabólicos como a diabetes mellitus ou sofrem de obesidade, e ainda expor como hábitos de vida podem interferir na infecção do SARS-CoV-2. Trata-se de um levantamento bibliográfico sobre os principais consensos e estudos sobre a COVID-19. Sabe-se que o vírus inicia o processo de infecção no trato respiratório superior para dar início ao ciclo de replicação viral. A diabetes mellitus é considerada um fator de risco de alta gravidade e mortalidade, uma vez que a hiperglicemia altera a resposta imunológica do indivíduo, resultando em maiores riscos de infecções. Igualmente, pessoas obesas possuem um risco significativo devido à alta produção de citocinas pró-inflamatórias e alterações funcionais das células $T$ que fazem ter uma resposta imunológica debilitada para conter a infecção. É essencial investir em medidas de prevenção de doenças, como a prática de atividades físicas e mudanças para bons hábitos alimentares com objetivo de melhorar a qualidade de vida e ter uma resposta imune eficaz, especialmente, no contexto pandêmico.

Palavras-chave: Coronavírus; SARS-CoV-2; metabolismo; inflamação.

\section{ABSTRACT}

In 2019, SARS-CoV-2 virus was discovered, causing a global health crisis. The 2020 pandemic, caused by the so-called "Coronavirus disease 2019" or COVID-19, spread rapidly around the world, impacting the global population and especially some groups at risk, such as patients with cardiovascular diseases, obesity and diabetes. This study aims to analyze and report the relationships between COVID-19 and patients with metabolic disorders such as diabetes mellitus or obesity, and also to expose how life habits can interfere with SARS-CoV-2 infection. This is a literature review on the main consensus and studies about COVID-19. It is known that the virus initiates the process of infection in the upper respiratory tract to begin the cycle of viral replication. Diabetes mellitus is considered a risk factor of high severity and mortality, since hyperglycemia alters the immune response of the subject, resulting in higher risks of infections. Also, obese people have a significant risk due to high production of pro-inflammatory cytokines and T-cell functional changes that cause a weakened immune response to contain the infection. It is essential to invest in disease prevention measures, such as the practice of physical activities and changes to good eating habits in order to improve life quality and to have an effective immune response especially in the pandemic context.

Keywords: Coronavirus; SARS-CoV-2; metabolism; inflammation. 


\section{INTRODUÇÃO}

No ano de 2002, no sudeste chinês, mais precisamente em Hong Kong, houve um surto de síndrome respiratória aguda grave (SARS) por um vírus, que posteriormente ficou conhecido como SARS-coronavírus (SARS-CoV). A princípio, tal enfermidade gerava acometimentos no trato respiratório inferior, engendrando uma pneumonia atípica potencialmente letal'.

Quase uma década após os quadros relatados de infecção por SARS-CoV, surge no mundo uma nova zoonose: a Síndrome Respiratória do Oriente Médio (MERS-CoV) que possuía como sintomas tosse e febre alta1.

No entanto, a trajetória dessas moléstias respiratórias não se restringiu a épocas retroativas, fazendo-se presente novamente em tempos atuais. Em 2019, foi encontrado um novo coronavírus zoonótico mais infectivo do que o SARS-CoV e MERSCoV, o SARS-CoV-2, sendo identificado pela primeira vez em Wuhan, na província chinesa de $\mathrm{Hubei}^{2}$.

Assim, desde 11 de março de 2020, vive-se a pandemia do novo coronavírus. Inicialmente, em 31 de janeiro de 2020, a Organização Mundial da Saúde (OMS) anunciou que listaria a infecção como uma emergência global e, em seguida, chamou a doença de COVID-193.

Os CoVs são os vírus RNA mais conhecidos pela literatura científica, sendo assim divididos em quatro gêneros: alfa-coronavírus, beta-coronavírus, gama-coronavírus e delta-coronavírus. Até o momento em que se tem ciência, foram localizados seis coronavírus humanos, os chamados HCoVs, que incluem: alfa-CoVs HCoVs-NL63 e HCoVs-229E e os beta-CoVs HCoVs-OC43, HCoVs-HKU1, SARS-CoV e MERSCOV4.

O espectro clínico da COVID-19 é amplo, indo desde um resfriado até uma grave pneumonia. Clinicamente, o início da doença expõe sintomatologia gripal simples, podendo evoluir para outras manifestações que estão presentes em outras doenças respiratórias como: febre, tosse seca, fadiga e dispneia. No entanto, em alguns casos, podem-se ter casos de hemorragia pulmonar, linfopenia grave, insuficiência renal e outros desdobramentos que variam de acordo com o estado de cada paciente ${ }^{2}$.

No parâmetro da COVID-19, sabe-se que vários fatores de risco estão associados a esta doença e a seu agravamento. Estudos comprovam que a existência de comorbidades aumenta em 2,4 vezes a probabilidade de morte por COVID-19 em comparação aos pacientes que mostram ausência de condições pré-existentes ${ }^{5}$. Assim, elencam-se como as comorbidades mais relevantes a hipertensão arterial, doenças cardiovasculares, diabetes e mazelas respiratórias, sendo essas consideradas fatores de risco para a fatalidade haja vista que corroboram para a piora do paciente e culminam em um tratamento mais tortuoso ${ }^{5}$.

Tais comorbidades relacionadas e suas condições de suscetibilidade podem estar ligadas à patogênese da COVID-19. Doenças crônicas compartilham características com doenças infecciosas, como o estado pró-inflamatório, atenuação do componente inato da resposta imune e baixa função imunológica, ao prejudicar macrófagos e a função linfocitária, o que pode corroborar em tornar os indivíduos mais suscetíveis a complicações da doença6. Sabe-se que a obesidade e suas condições associadas, principalmente o diabetes do tipo 2, crescem exponencialmente no mundo. É digno de nota que são enfermidades graves que caminham juntas até certo ponto, com grande colaboração de maus hábitos de vida. Nesse contexto, é fundamental apreender que os novos conhecimentos da fisiopatologia da doença estão sendo úteis na implementação de medidas de prevenção mais eficazes e nas pesquisas para o desenvolvimento de novos tratamentos, buscando a erradicação da doença7.

\section{OBJETIVOS}

Este trabalho tem como objetivo analisar e descrever as relações existentes entre a pandemia de COVID-19 e os quadros de diabetes e de obesidade, e ainda relatar a influência do estilo de vida na infecção por SARS-CoV-2.

\section{MÉTODOS}

Trata-se de uma revisão de literatura, em que se utilizaram as seguintes bases de dados: SciELO, Pubmed e ClinicalKey. Foram buscados artigos científicos que exploram diferentes comorbidades metabólicas e sua relação com a infecção pelo novo coronavírus, com objetivo de aperfeiçoar e integrar o conhecimento.

As palavras-chave utilizadas foram: COVID19, obesidade, diabetes mellitus, metabolismo e ECA-2. Para a seguinte revisão literária, foi dada preferência para os artigos mais atuais sobre cada assunto abordado, sendo incluídos apenas os trabalhos cujo texto fosse citado nas bases de dados de pesquisas referenciadas. 


\section{ESTRUTURA DO VÍRUS}

Os Coronavírus foram descritos primeiramente no ano de 1966 por Tyrell e Byone, que cultivavam os vírus de pacientes com resfriados comuns $^{8}$. Eles representam um grupo de vírus de RNA envelopado que possuem sentido positivo, pertencente à subfamília Coronavirinae, família Coronaviridae, sendo parte da ordem Nidovirales ${ }^{9}$.

Sob microscopia eletrônica, eles são visualizados como círculos com espículas que se exteriorizam e geram projeções em um formato de coroa, fato que origina a expressão "corona" (significa coroa em latim)2. Os CoVs são classificados em gêneros $\alpha, \beta$, ye $\delta$, dos quais somente os CoVs $\alpha$ e $\beta$ infectam humanos ${ }^{10}$.

O genoma dos CoVs possui uma variação quanto ao número de estruturas de leitura aberta, os chamados ORFs. Dois terços do RNA viral, primordialmente na primeira ORF traduzem as poliproteínas pp1a e pp1b, que, por sua vez, são capazes de codificar 16 proteínas não-estruturais (NSP). Sendo assim, as demais ORFs ficam responsáveis por realizar a codificação de proteínas acessórias e estruturais ${ }^{11}$.

A parte que resta do genoma do vírus codifica, então, quatro proteínas estruturais essenciais: proteínas Spike, membrana, envelope e nucleocapsídeo ${ }^{11}$. Com isso, o COVID-19 torna-se uma partícula esférica ou pleomórfica bastante complexa ${ }^{9}$.

\section{Proteína Spike}

A proteína Spike, ou simplesmente proteína $S$, é uma proteína de fusão viral de classe 1 que proporciona a adesão ao hospedeiro e a fusão das membranas virais e celulares. Dessa maneira, a proteína $S$ é capaz de determinar a gama de hospedeiros e o tropismo celular, sendo o principal foco dos anticorpos neutralizantes e o alvo em projetos de vacinação ${ }^{10}$.

Proteína de Membrana

A glicoproteína de membrana (proteína M) é o elemento que aparece na estrutura em maior proporção, sendo essa que realiza a passagem da bicamada cerca de três vezes. Com esse trajeto, tal componente deixa um pequeno domínio $\mathrm{NH} 2$ terminal fora do vírus e um longo terminal $\mathrm{COOH}$ dentro do vírus, ou seja, um domínio citoplasmático ${ }^{9}$.

\section{Proteína do Envelope}

A proteína do envelope é a menor das principais proteínas estruturais, sendo também a mais misteriosa em termos de composição. No ciclo de replicação, é expressa de forma abundante no interior da célula infectada, porém só uma pequena porção é incorporada ao envelope do vírion. Apesar de enigmática, foram determinadas as principais funções dessa estrutura: participação da montagem viral, liberação de vírions e patogênese do vírus ${ }^{12}$.

Proteína do Nucleocapsídeo

A proteína nucleocapsídica forma junto com - RNA do genoma uma estrutura de capsídeo helicoidal que se encontra dentro do envelope viral. Possui como funções um papel na formação complexa do genoma viral e facilita a interação da proteína $M$ que é imprescindível durante a montagem do vírion, auxiliando na eficiência da transcrição viral ${ }^{13}$.

Até o presente momento, mesmo que sejam conhecidos componentes estruturais do vírus, não existe tratamento específico completamente eficaz contra a pandemia de COVID-19. No entanto, compreender e saber identificar as bases de sua patogenicidade são passos cruciais para trilhar o caminho da descoberta de um tratamento ou prevenção efetivos.

\section{INFECÇÃO POR SARS-COV-2}

$O$ vírus inicia o processo de infecção no trato respiratório superior, invadindo as células epiteliais do mesmo para dar início ao ciclo de replicação viral. OSARS-CoV-2 penetra nas células através da proteína $S$ que se localiza na superfície do coronavírus. Essa proteína tem a capacidade de se ligar à enzima conversora de angiotensina 2 (ECA2), que é uma proteína de membrana tipo I expressa no pulmão, coração, rim e intestino. A proteína $S$ possui duas subunidades, a S1 que determina o alcance do vírus e o seu tropismo celular até o domínio de ligação ao receptor ( $R B D$ ), e a subunidade $\mathrm{S} 2$, a qual intermedia a fusão da membrana da célula com o vírus através dos de dois domínios heptad repeat 1 (HR1) e HR214. Após a fusão da membrana, o genoma de RNA viral é liberado no citoplasma da célula e, então, passa a produzir proteínas não estruturais, e formar o complexo de replicação e transcrição, o qual começa a replicar e sintetizar RNAs virais. Assim, desencadeia-se a produção de proteínas acessórias e proteínas estruturais. Logo em seguida, os RNA genômicos recém-formados se juntam às proteínas nucleocapsídicas e ao envelope glicoproteico e formam uma nova partícula viral. Finalmente, as 
novas vesículas que agora contém o vírus se fusionam com a membrana plasmática de outras células saudáveis do hospedeiro para liberar os novos vírus prontos $^{11}$.

A perda de ECA2 devido à entrada do SARSCoV-2 acaba gerando consequências diversas no corpo humano, já que ela é peça chave para a conversão da Angiotensina ${ }^{2}$ e encontra-se expressa em muitos órgãos. A resposta deletéria é observada em especial no pulmão, onde a agressão às células que possuem expressão da ECA ${ }^{2}$ pode contribuir para a inflamação generalizada, desconforto respiratório e possível fibrose das áreas afetadas do pulmão observada em pacientes infectados por SARS-CoV$2^{15}$.

\section{DIABETESMELLITUS}

Diabetes é uma doença determinada pela hiperglicemia associada a síndromes metabólicas como resistência insulínica, obesidade, dislipidemia e hipertensão, além de eventos, como ativação e disfunção endotelial ${ }^{16}$. A hiperglicemia altera a resposta imunológica do indivíduo, resultando em maiores riscos de infecções por diversos patógenos como também aumenta a gravidade destas. Frente ao cenário de pandemia por SARS-CoV-2, a diabetes mellitus é considerada como fator de risco, aumentando a gravidade e mortalidade, assemelhando-se tal risco às infecções anteriores por SARS-CoV, em 2002 e MERS em $2012^{17}$.

Entre as doenças adjacentes associadas a hospitalizações e internações por SARS-CoV-2 na China e Itália, a diabetes obteve destaque. Um estudo na China evidenciou que entre 168 casos fatais de pneumonia por SARS-CoV-2 ocorridos em 21 hospitais de Wuhan entre 21 e 30 de janeiro de 2020, $75 \%$ eram homens, com idade média de 70 anos, sendo diabetes relatada em 42 pacientes, $25 \%$ dos $\operatorname{casos}^{18}{ }^{19}$. Após doenças cardíacas metabólicas, diabetes é a segunda enfermidade mais incidente em pacientes com COVID-19 20, 21,22 .

Segundo a American Diabetes Association (2020), pacientes com diabetes são mais propensos a apresentarem sintomas e complicações mais graves de COVID-19, principalmente os que não controlam bem a doença ${ }^{23}$. No entanto, o risco de infecção por SARS-COV-2 é aproximadamente o mesmo comparado à população em geral e inclusive com uma taxa ligeiramente menor ${ }^{24}$.

Ressaltam-se neste contexto as consequências deletérias da diabetes no funcionamento pulmonar como alterações parenquimatosas, vasculares e das membranas alveolares, uma vez que estudos demonstraram o espessamento dos capilares basais alveolares e das lâminas epiteliais nos indivíduos diabéticos ${ }^{25},{ }^{26}, 27$. Ademais, a debilidade da função pulmonar nestes pacientes pode aumentar, como evidenciado por Davis e colaboradores (2004), ao observar 125 pacientes com diabetes tipo 2 durante 7 anos, relatando declínio de $71 \mathrm{~mL} /$ ano no volume expiratório forçado $(\mathrm{VEF} 1)^{28}{ }^{29}$. Tais alterações fisiopatológicas quando avançadas podem propiciar o acometimento pulmonar conjuntamente com a síndrome respiratória aguda pelo coronavírus.

\section{Sistema Imunológico e Diabetes}

Quadro hiperglicêmico modifica as respostas imunológicas perante as infecções, promovendo alteração na liberação de citocinas por macrófagos e células $T$, que interferem no recrutamento de linfócitos concomitantemente 30 . Além disso, em ambos os tipos de Diabetes, tipo $1 \mathrm{e}$ tipo 2 , há redução da produção de interferon - alfa (IFN- $\alpha$ ), O qual possui propriedade antiviral ${ }^{31}$.

Observa-se nos pacientes diabéticos uma acentuação da resposta pró-inflamatória, como a mediada pela liberação de interleucina - 1 (IL-1), IL-6 e fator de necrose tumoral - alfa (TNF- $\alpha$ ), e um retardo na ativação de células $T$ helper (Th1). Tais alterações imunológicas propiciam maiores complicações de COVID-1932, ${ }^{33}$.

\section{Diabetes e SARS-CoV-2}

Os processos endoteliais (ativação e disfunção endotelial) em nível pulmonar, tanto derivados da infecção por SARS-CoV-2 quanto da diabetes, corroboram para o espessamento da membrana basal. Consequentemente, torna-se deficitária a troca alveolar de gás pela barreira sangue-gás, a qual também possui relevância em resposta aos danos nos pneumócitos decorrentes da liberação de citocinas na COVID-19. Como resultado, as alterações causadas pelo próprio vírus e pela diabetes possivelmente aceleram a progressão da infecção pelo SARS-CoV-2 associada a maiores chances de necessidade de ventilação mecânica e maior morbidade e mortalidade ${ }^{28,},{ }^{34},{ }^{16}$.

A relação entre diabetes e coronavírus atualmente também é exemplificada pela ECA2, que atua como transdutora de vias metabólicas reguladoras em vários sistemas no organismo, além de ter sido identificada como o sítio de ligação para o vírus adentrar nas células do hospedeiro ${ }^{35}$. Ambas as 
isoformas de ECA2, ECA2 transmembranar e sua forma solúvel (sECA2), servem de sítio de ligação para a proteína spike (S1) do coronavírus auxiliado pela TMPRSS2 (serina protease transmembranar 2), adentrando nas células do trato respiratório ${ }^{35}$.

Até o momento, acredita-se que ocorra uma hiperexpressão da ECA2 com o uso de fármacos inibidores de ECA2 (iECA) e bloqueadores do receptor de angiotensina (BRAs), os quais são frequentemente utilizados na diabetes devido a sua associação com hipertensão e albuminúria. Por meio da super regulação desta enzima, o uso de tais fármacos poderiam facilitar a entrada do SARS-CoV-2 nos pneumócitos, induzindo a síndrome respiratória aguda e, posteriormente, seu agravamento32. Resultados de biópsia do duodeno de pacientes tratados com iECA demonstraram aumento da transcrição do RNA-m de ECA2, corroborando tal hipótese36. Entretanto, não é bem elucidada a relação dessa enzima com o controle dos níveis glicêmicos ${ }^{35}$.

Além disso, a resistência insulínica e a hiperglicemia intensificam reações de glicosilação e seus produtos, promovendo eventos inflamatórios, como estímulo de citocinas pró-inflamatórias, moléculas de adesão e estresse oxidativo, que conjuntamente podem contribuir para o quadro infeccioso por coronavírus ${ }^{37},{ }^{38}$. Por outro lado, não há evidências de aumento das infecções nos indivíduos com diabetes do tipo 135 .

\section{Fármacos utilizados durante a Pandemia}

Não há comprovação científica de efeitos maléficos ou benéficos em relação à suspensão de fármacos que interferem a atuação da ECA2 como inibidores da ECA2 ou bloqueadores dos receptores de angiotensina associados à infecção por coronavírus $^{35}$. De acordo com a Sociedade Brasileira de Diabetes, Conselho de Hipertensão da Sociedade Europeia de Cardiologia e do Departamento de Hipertensão Arterial da Sociedade Brasileira de Cardiologia, infere-se a manutenção desses medicamentos adjunto ao controle dos níveis glicêmicos e monitoramento deste durante a pandemia.

O controle glicêmico deve ser monitorado. pois o desequilíbrio pode interferir na ligação do vírus na ECA2 assim como a intensidade da resposta imune ao vírus ${ }^{39}$.

\section{OBESIDADE}

A obesidade (IMC maior ou igual a $30 \mathrm{~kg} / \mathrm{m} 2$ ) é uma condição patológica definida pelo acúmulo excessivo de tecido adiposo corporal, capaz de exercer uma influência negativa no funcionamento do organismo de forma sistêmica. Ela se faz presente na síndrome metabólica, junto a outras comorbidades como resistência à insulina (hiperglicemia), hipertensão arterial sistêmica e dislipidemia. Estudos realizados em vários países demonstram que a obesidade se encaixa entre uma das principais comorbidades encontradas em pacientes acometidos pela COVID-1940, 11,42 . Além disso, há uma clara comprovação de pior prognóstico, maior mortalidade e severidade da COVID-19 em pacientes nessas condições ${ }^{43}$.

Experiências atuais europeias indicam casos mais graves de COVID-19 associados à obesidade nas faixas etárias mais jovens. De uma forma geral, pacientes obesos gravemente enfermos $(5 \%$ das infecções) necessitam de cuidados intensivos, representando, assim, uma série de desafios para os sistemas de saúde, como necessidade de mais leitos hospitalares bariátricos, intubações mais desafiadoras, maior dificuldade para obter diagnósticos por exames de imagem devido aos limites de peso nas máquinas, além de maior dificuldade de posicionamento e transporte pela equipe de enfermagem. Mesmo fora das unidades de terapia intensiva, pessoas com obesidade já estão vivendo mais isoladas, evitando o contato social, pois já estão estigmatizadas e apresentam índices maiores de depressão44.

Um estudo brasileiro de Rezende e colaboradores (2020) pesquisou a incidência de uma série de comorbidades em pacientes com COVID-19 (dentre elas doenças cardiovasculares, diabetes, doença respiratória crônica, hipertensão, câncer, derrame, obesidade, doença hepática crônica e asma de moderada a severa, além de maus hábitos de vida como o tabagismo) e sua relação com o estado grave da infecção pandêmica. Quando comparada às outras comorbidades, a obesidade representa no Brasil $22 \%$ dos fatores de risco para mau prognóstico de COVID-19 em pacientes com menos de 65 anos de vida, e 22,7\% para pacientes acima desta idade ${ }^{45},{ }^{46}$. Estudos norte-americanos também relatam que a obesidade estava presente em $25 \%$ dos casos de óbito por COVID-1947. Apesar de o fato dessa comorbidade não ter sido relatada nos estudos iniciais feitos em Wuhan, pesquisas comparativas em Nova York e Nova Orleans (cidade com alta prevalência de obesidade nos EUA) sugerem que a obesidade tornou-se um importante fator de mau 
prognóstico48. Essa associação entre obesidade e mau desfecho de síndromes gripais já tem se mostrado presente desde a epidemia de $\mathrm{H} 1 \mathrm{~N} 1^{49}$.

Obesidade e Alterações Imunológicas

De uma forma geral, a inflamação decorrente da obesidade pode ser desencadeada por vários mecanismos moleculares que explicam as suas complicações. As consequências estão relacionadas a um quadro de síndrome metabólica, comprometendo o sistema cardiovascular e multiplicando os fatores de risco para uma maior severidade da COVID-1950.

A obesidade é caracterizada por um processo inflamatório crônico de baixo grau, com elevação da produção de citocinas pró-inflamatórias, como TNF- $\alpha$, IL-1 e IL-6, além de alterações funcionais das células $T$ em decorrência do prejuízo do metabolismo (como hiperglicemia e dislipidemia). Com relação à COVID-19, dados iniciais apontam que, para conter essa infecção, faz-se necessário que o organismo possua uma resposta imunológica ampla e coordenada, na qual se faz presente a ativação de células $T$, com migração de diferentes células imunes (linfócitos, neutrófilos e macrófagos), e produção paralela de uma série de citocinas51. Sendo assim, o combate à infecção é prejudicado nos indivíduos obesos. Ademais, a tempestade de citocinas liberadas nesses pacientes tem o potencial de causar danos graves no tecido pulmonar, contribuindo para a maior severidade da COVID-19 e levando a uma maior necessidade de suporte ventilatório mecânico durante a hospitalização. Sabe-se ainda que tanto diabéticos quanto obesos possuem alterações na liberação e na ação de IFN, citocina importante para coordenar a resposta contra a infecção viral ${ }^{43}$.

Outro mecanismo dessa relação entre sistema imunológico e obesidade se dá através da leptina, uma proteína produzida pelos adipócitos que atua como um fator de sinalização entre o tecido adiposo e o sistema nervoso central, regulando a ingestão alimentar, o gasto energético e, consequentemente, a massa corporal. Além disso, essa proteína aparentemente possui efeitos específicos na função dos linfócitos $T$, que se dão pela regulação da proliferação de células envolvidas na resposta imune, tanto inata quanto adquirida. Com isso, a leptina aumenta a produção de linfocinas próinflamatórias ${ }^{52}$. Sabe-se que na obesidade há um quadro de hiperleptinemia (excesso de leptina circulante) e resistência à leptina, o que contribui com o desajuste na reação inflamatória de combate à COVID-1943.

Outra proteína de importante associação com a obesidade, secretada especificamente pelo adipócito, mas também expressa em monócitos e macrófagos, é a resistina. Ela tem como função induzir à expressão de algumas moléculas de adesão e de algumas citocinas como TNF- $\alpha$ e IL- 6 , mas sua maior relevância no que diz respeito ao maior risco para COVID-19 é a sua correlação com a doença arterial coronariana. Essa proteína é considerada um marcador inflamatório em humanos, com grande potencial pró-inflamatório, estando também associada à resistência insulínica e à síndrome metabólica50.

\section{ECA-2: Um Forte Elo entre Obesidade e COVID-19}

Além dessa associação com as perturbações imunológicas, algumas revisões de literatura destacam que o tecido adiposo pode ser um depósito viral, uma vez que apresenta a ECA2, considerada a principal porta de entrada do vírus SARS-CoV-2. Sendo assim, é possível ainda explorar a possibilidade de uma infecção diretamente através do tecido adiposo e seu impacto na produção de citocinas próinflamatórias, além do espalhamento do vírus pelo corpo a partir dos adipócitos43. Alguns experimentos realizados em ratos evidenciaram que a obesidade causa aumento da expressão da proteína ECA2, com a qual as proteínas $S$ do coronavírus interagem ${ }^{53}$.

Sabe-se ainda que essa enzima degrada a Angiotensina II, que é uma substância potentemente hipertensora, convertendo-a em angiotensina 1-7. Esta última, por sua vez, atua em receptores específicos chamados Mas-R ("Mas Receptor"), promovendo ações anti-inflamatórias, anti-fibróticas e vasodilatadoras. Apesar do aumento da expressão de ECA2 evidenciado em ratos obesos, tal observação foi feita em quadros agudos de hiperglicemia e obesidade, enquanto esses quadros crônicos mostram um efeito oposto (redução da ECA2) e, consequentemente, falhas na conversão de angiotensina II em angiotensina 1-7. Desta forma, isso parece favorecer anormalidades vasculares e inflamatórias nesses indivíduos, afetando inclusive a proteção pulmonar $^{43}$.

Outras Alterações Complicadoras da COVID-19 Advindas da Obesidade

Existem relatos que enfatizam a presença de mudanças no comportamento respiratório diante do quadro de obesidade. Muitos estudos demonstram 
uma associação entre excesso de peso ou ganho de peso com disfunção pulmonar, provavelmente devido à diminuição da complacência da parede torácica ou inatividade ${ }^{54,55,56}$. Além disso, observase uma redução de volume de reserva expiratório, o que está associado ao acúmulo de gordura abdominal sobre a caixa torácica, e aumento da resistência de vias aéreas. Desta forma, o comprometimento pulmonar próprio da COVID-19 se soma a esse quadro, ocasionando um mau prognóstico ${ }^{57}$.

Outro fator intimamente relacionado à obesidade é a injúria hepática. De acordo com dois estudos, a incidência de elevação de enzimas hepáticas em pacientes hospitalizados com COVID19, principalmente AST e ALT, varia de $14 \%$ a $53 \%$, sendo mais comum em pacientes masculinos e em estágios mais graves da doença. Em aproximadamente $10 \%$ dos casos, eleva-se ainda discretamente a bilirrubina total. Além disso, a redução da albumina tem se mostrado como um marcador de maior gravidade ${ }^{58,59}$.

Em alguns artigos, também são mencionados problemas na cascata de coagulação relacionados à obesidade. Quando esse quadro é relacionado à COVID-19, sabe-se que essa infecção viral possui forte impacto na coagulação sanguínea, provocando frequentes eventos relacionados à hipercoagulabilidade, que podem estar relacionados à formação de D-dímeros e fibrinogênio ${ }^{43}$.

\section{Influência do Fator Nutricional na COVID-19}

Sabe-se também que o estado nutricional interfere diretamente na resposta imunológica do indivíduo, e a inadequação nutricional é um grande fator da obesidade. De acordo com os estudos de Berthon e Wood (2015), o papel da nutrição na saúde respiratória é de suma relevância, e determinadas dietas representam fatores de risco para o desenvolvimento de doenças pulmonares. Os autores relatam os benefícios de algumas dietas ricas em alimentos com compostos antioxidantes, vitaminas A, D, E, C, flavonóides, ácidos graxos poliinsaturados, ômega 3 e minerais, como, por exemplo, a dieta mediterrânea. Esses estudos demonstram evidências do efeito positivo desses nutrientes em pacientes com doença pulmonar obstrutiva crônica (DPOC) e asma, bem como os efeitos negativos de dietas ocidentais, ricas em grãos refinados, carnes curadas, doces e pobres em fibras e nutrientes essenciais, além dos eventos inflamatórios da obesidade e sua ligação a estas condições, como já foi explicitado60,61.

\section{INFLUÊNCIA DO ESTILO DE VIDA}

De acordo com um estudo publicado no The Lancet por Guthold e colaboradores (2018), em que foi feito um ensaio com participantes de 18 anos de idade ou mais em 168 países para estimar a prevalência de atividade física insuficiente na população adulta, $53,3 \%$ das mulheres e $40,4 \%$ dos homens brasileiros não praticavam atividade física suficiente em 2016. Ademais, dados do IBGE (2013) revelam que os estados de São Paulo e Rio de Janeiro, por exemplo, possuem índices elevados de pessoas com circunferência de cintura aumentada, sendo São Paulo o maior ( $43,1 \%$ de indivíduos) e o Rio de Janeiro o terceiro ( $41,6 \%$ de indivíduos). Esses altos valores de circunferência da cintura são relacionados a diversos riscos, como aumento da pressão arterial, diabetes e dislipidemia, que são preditores de problemas cardiovasculares e claramente fatores de risco para a COVID-19. Os dados de pressão arterial apontam que $22,3 \%$ da população brasileira acima de 18 anos possui hipertensão arterial sistêmica. Deste modo, deve-se procurar uma resposta que explique o porquê de uma população ainda tão sedentária, mesmo com incentivos à prática de atividades físicas ${ }^{62},{ }^{63}$.

Outro fator relacionado ao maior risco de severidade da COVID-19 devido a maus hábitos de vida diz respeito ao tabagismo. Os primeiros estudos publicados sobre a associação entre fumar e se infectar e/ou progredir para complicações respiratórias graves em pacientes com essa infecção vieram já da China, que foi o primeiro epicentro da pandemia, evidenciando uma clara correlação entre tabagismo e mau prognóstico de COVID-19. A epidemia do tabagismo matou cerca de 100 milhões de pessoas no século XX64, e mata até hoje cerca de oito milhões de pessoas por ano no mundo, sendo somente no Brasil 156 mil óbitos por ano ocasionados pelos hábitos tabagistas65. Além disso, em um país tão carente de recursos para atender a questões emergenciais de saúde, como esta causada pela infecção pelo SARS-CoV-2, aproximadamente 57 bilhões de reais são consumidos anualmente em perda de produtividade e gasto direto com tratamento de fumantes66.

$O$ etilismo também tem sido abordado por pesquisadores que alertam para alguns fatores de risco relacionados à COVID-19. Alguns estudos preliminares realizados em países como China, Reino Unido e Espanha apontam um aumento nos quadros 
de ansiedade, depressão e menor bem-estar mental em relação ao habitual durante o período de isolamento social, e o consumo excessivo de álcool possui íntima associação com o papel da ansiedade e do estresse ${ }^{67}$. Adicionalmente, sabe-se que esse uso abusivo de álcool reduz, em diferentes aspectos, a atividade do sistema imunológico, potencializando, assim, as complicações da COVID-1968.

\section{Atividade Física e COVID-19}

Estudos indicam que exercícios físicos de moderada intensidade têm efeitos positivos nas respostas do sistema imunológico contra infecções respiratórias virais. Após a prática de atividade física, é detectado um aumento na contagem de neutrófilos e de células natural killer (NK) e nas concentrações salivares de IgA. A atividade física moderada também aumenta os níveis de hormônios como cortisol e adrenalina e reduz a inflamação excessiva, levando ao aumento da reação imunológica contra infecções virais através da alteração nas respostas das células Th1/Th2. 0 impacto de um estilo de vida sedentário, embora pareça ser menor em crianças e adultos jovens, é decisivo em pessoas com idade avançada (acima dos 60 anos), obesidade, diabetes mellitus, hipertensão arterial sistêmica, doença cardiovascular, tabagistas e portadores de DPOC. Desta forma, chega-se à conclusão de que a atividade física prévia e uma boa aptidão cardiorrespiratória, obtidos através do treinamento aeróbico de intensidade moderada, são fatores que conferem imunoproteção em pacientes infectados pelo SARS-CoV- $2^{69}$.

É indubitável que os programas de atividade física e saúde não atingem uma parcela significativa da população. Entre alguns pontos que podem ser revistos, estão a divulgação e o incentivo à prática de exercícios físicos e a orientação profissional. É essencial que a população conheça os benefícios da prática regular de atividade física e seja incentivada a sempre se movimentar conhecendo o porquê dessa necessidade. Além disso, a falta de supervisão profissional pode tornar o exercício físico prejudicial à saúde do praticante, pois movimentos errados podem ocasionar lesões e atividades mal elaboradas podem ser ineficientes para promoção de saúde e qualidade de vida ${ }^{52}$.

A impossibilidade de contato social e até mesmo de frequentar academias e outros centros de atividade física tem impulsionado uma nova organização no modo de se exercitar através de adaptações tanto dos educadores físicos quanto dos alunos a uma nova rotina, desta vez de forma virtual. Deste modo, espera-se que esse novo modo de vida ativo atenue as dificuldades do distanciamento social, não somente pelos benefícios físicos e psicoemocionais, mas também pelo convívio social ainda que virtualmente. Desta forma, pode-se estimular o ser humano a manter seu bem-estar e, por conseguinte, sua saúde ${ }^{70}$.

\section{Tabagismo e COVID-19}

A relação entre tabagismo e COVID-19 já se inicia pelo maior risco de contaminação através do próprio ato de levar os produtos do tabaco à boca sem a adequada higienização das mãos e/ou dos aparatos, especialmente através dos narguilés, muito populares entre a população jovem, e dos dispositivos eletrônicos para fumar (DEFs). De acordo com a literatura, doenças como influenza, herpes labial e tuberculose são transmitidas por bocais do narguilé, e as características que possibilitam o compartilhamento de DEFs também contribuem para a contaminação pelo vírus SARS-CoV-2. Além disso, o fumante pode sofrer queimaduras ao acender o cigarro ou ao manusear o narguilé após higienizar as mãos com álcool em gel, uma vez que esse produto é altamente inflamável. Em relação aos danos pulmonares, o tabagismo está relacionado à bronquiolite respiratória (geralmente assintomática), apresentando vários tipos de pneumonias, além de bronquite crônica, enfisema pulmonar, tuberculose e cânceres de pulmão. Desta forma, isso ocasiona um declínio da função pulmonar, que pode tornar a infecção pela COVID-19 fatal ${ }^{71}$.

De acordo com uma análise do desfecho de pacientes com DPOC e/ou hábitos tabagistas realizada em uma revisão sistemática com dados de pesquisas publicadas até março de 2020, os fumantes tiveram um risco de mortalidade $38,5 \%$ maior do que os não fumantes, e o risco para complicações severas relacionadas à COVID-19 em fumantes foi $45 \%$ maior do que aquele observado entre os não fumantes. Em outra revisão de literatura seguida de metanálise, em que os autores fizeram buscas até 28 de abril e analisaram 19 estudos sobre essa mesma temática (sendo 1 da Coréia, 2 dos Estados Unidos e 16 da China), foi indicada uma probabilidade de aproximadamente o dobro dos pacientes com história de tabagismo (fumantes ou ex-fumantes) evoluírem para um desfecho mais desfavorável quando comparada às chances dos não fumantes. Esses autores também realizaram uma 
análise de sensibilidade incluindo apenas os fumantes atuais (cinco estudos dos 19 inicialmente selecionados) e encontraram um resultado semelhante ${ }^{66}$.

Estudos realizados por Smith e colaboradores (2020) destacaram que os pulmões dos fumantes apresentam níveis elevados de ECA2. Sabe-se que essa enzima é expressa em uma subpopulação de células secretoras que se expandem em resposta à exposição à fumaça, estimulando o gene da ECA2 por interferon, que é expresso quando há infecções virais ${ }^{72},{ }^{73}$.

Sabe-se ainda que a prática tabagista tem relação direta com a disfunção endotelial e a elevação da concentração dos radicais livres, da mesma forma que infecções como a COVID-19. Desta forma, acredita-se que essa infecção pandêmica possa ocasionar danos ao endotélio previamente lesionado do fumante, e observa-se que a cessação do tabagismo melhora significativamente a função endotelial. Além disso, pacientes infectados pelo SARS-CoV-2 apresentam níveis elevados de proteína $C$ reativa $(P C R)$ e dímero- $D$, e esses marcadores de diagnóstico de trombose encontram-se também alterados em fumantes. Um estudo observou quadro de coagulação intravascular disseminada (CID) em $71 \%$ dos óbitos por COVID-19 comparado com 0,4\% dos sobreviventes. Somado a isso, níveis mais altos de dímero-D (acima de $1 \mu \mathrm{g} / \mathrm{L}$ ) na admissão aumentam em 18 vezes a chance de evolução para óbito. O mecanismo dessas complicações ainda é desconhecido, mas elas sugerem a relevância do impacto do tabagismo no endotélio e no prognóstico da COVID-1971.

Apesar de todos esses fatos evidenciarem a suma importância de abandonar o fumo e inibir sua iniciação, torna-se uma missão grandemente desafiadora estimular essa decisão diante de uma situação de estresse, incerteza quanto ao futuro e de isolamento social em casa. Para tal, faz-se necessário também reconhecer e atuar na tentativa de minimizar a interferência da indústria do tabaco nas políticas públicas, reforçando o impacto negativo dos gastos em saúde atribuíveis ao uso do tabaco66.

\section{Etilismo e COVID-19}

Com base em uma revisão bibliográfica realizada por Rehm e colaboradores (2020), observam-se duas hipóteses principais acerca da relação entre a pandemia de COVID-19 e as alterações no padrão de consumo de álcool. A primeira sugere um aumento no consumo de álcool em algumas populações devido ao aumento do sofrimento psicológico desencadeado pela interação de dificuldades financeiras, isolamento social e incerteza sobre o futuro. Um estudo chinês sobre a pandemia de SARS em 2003, realizado com 800 moradores de Hong Kong, mostrou que $6,8 \%$ dos que consumiam álcool $(4,7 \%$ dos homens e $14,8 \%$ das mulheres) relataram um aumento deste consumo um ano após essa pandemia. A outra hipótese, baseada em evidências de pesquisas sobre políticas de controle do álcool, prevê reduções do consumo de álcool e dos problemas atribuíveis a ele devido a fatores relacionados à disponibilidade física e ao acesso ao álcool no sentido financeiro. Como crises do porte da pandemia de COVID-19 são geralmente associadas ao desemprego e à redução das jornadas de trabalho, levando à diminuição da renda para grande parte da população, é natural ter-se como consequência a redução do consumo de álcool ${ }^{68}, 74$.

Com relação à influência do etilismo no desfecho da COVID-19, estudos apontam que indivíduos alcoolistas apresentam maior incidência de sepse no prognóstico de infecções de forma geral. A resposta imunológica pode estar comprometida nesses pacientes, ocorrendo alterações nos mecanismos de defesa primários e comprometimento da imunidade celular e humoral. Além disso, a quimiotaxia dos granulócitos e os níveis de secreção de TNF encontram-se também reduzidos. Quando há dependência de bebida alcoólica associada a deficiências nutricionais e desequilíbrios sociais, como infelizmente é comum, pode ser desencadeada uma debilidade orgânica que facilita o surgimento de infecções e, assim, a transmissibilidade tão alta do SARS-CoV-2 é ainda maior nesses indivíduos ${ }^{75}$.

De acordo com estudos experimentais realizados por Andrade e colaboradores (2008), o etanol é capaz de prejudicar a atividade fagocitária dos macrófagos. Após a administração de altas doses de etanol em camundongos, observou-se uma deficiência global na produção de IL-6 e óxido nítrico (NO) por macrófagos peritoneais em decorrência de estímulos com lipopolissacarídeo (LPS). Além disso, o álcool é conhecido por alterar os níveis de citocinas no plasma e em diversos tecidos, incluindo fígado, pulmão e cérebro. Estudos realizados por Goral e colaboradores (2008) demonstraram ainda que o tratamento agudo com etanol in vivo ou in vitro inibe a produção de citocinas pró-inflamatórias em resposta a uma variedade de compostos microbianos. Somando todos esses agravantes, 
diante da pandemia de COVID-19, torna-se clara a forte tendência para um mau prognóstico nesses pacientes ${ }^{76}, 77,78$.

Diante de todos esses fatos, é de suma importância que sejam postos em prática projetos de prevenção ao abuso de álcool baseados nas normas sociais, buscando estimular estilos de vida mais saudáveis. Para isso, devem ser alicerçadas ações direcionadas à saúde pública na área de prevenção ao consumo abusivo de álcool durante a pandemia, tendo a mídia como veículo e agente potencializador $^{67}$.

\section{CONSIDERAÇÕES FINAIS}

O presente artigo discorre sobre os aspectos etiológicos da infecção por SARS-CoV-2 e sua correlação com algumas comorbidades diante da vigente pandemia. Segundo estudos originais e meta análises, destacam-se diversas comorbidades associadas à síndrome metabólica como doenças cardiovasculares, diabetes, dislipidemia e obesidade que apresentam incidência significativa em pacientes acometidos pela COVID-19, sendo classificados como fatores de risco. Tais comorbidades impactam no curso clínico com piora de prognóstico, mortalidade e complicações severas. Diabetes e obesidade possuem comprometimento sistêmico, afetando a resposta imunológica do indivíduo, exacerbando o quadro inflamatório associado à produção deficiente de IFN, o que incapacita a defesa do organismo frente à infecção viral. Além disso, ambas comorbidades modificam a expressão de ECA2. No que tange à diabetes, embora a ECA2 tenha função regulatória no equilíbrio da glicose, até o momento não fora bem elucidado se os fármacos que interferem na atuação desta, causam malefício ou benefício na infecção por coronavírus. Desse modo, os pacientes diabéticos não devem suspender sua medicação e devem controlar seus níveis glicêmicos. Em relação à obesidade, a elevação da leptina auxilia na intensificação da inflamação. Também se observa comprometimento pulmonar, assim como relatado na diabetes, além de fatores pró-trombóticos que agravam os danos vasculares característicos da infecção por coronavírus.

$O$ estilo de vida exerce também forte influência perante as infecções por SARS-CoV-2, uma vez que uma boa parcela da população não pratica atividade física, é tabagista e etilista. Como reflexo do isolamento e da falta de estímulo de hábitos saudáveis associados à redução de interação social, a saúde mental dos brasileiros deve ser posta em evidência já que todo esse contexto propicia o agravamento de ansiedade e depressão, também já relacionadas à COVID-19.

\section{REFERÊNCIAS}

1. de Wilde AH, Snijder EJ, Kikkert M, van Hemert MJ. Host Factors in Coronavirus Replication. In Springer C, editor. Roles of Host Gene and Non-coding RNA Expression in Virus Infection.; 2017. 1-42.

2. Lima CMAdO. Informações sobre o novo coronavírus (COVID-19). Radiol Bras. 2020 Abril; 53(2):V-VI.

3. Strabelli TMV, Uip DE. COVID-19 e o Coração. Arquivos Brasileiros de Cardiologia. 2020 Abril; 144(4):598-600.

4. Wu D, Wu T, Liu Q, Yang Z. The SARS-CoV-2 outbreak: What we know. International journal of infectious diseases. 2020 Maio; 94:44-48.

5. Espinosa OA, Zanetti AdS, Antunes EF, Longhi FG, de Matos TA, Battaglini PF. Prevalence of comorbidities in patients and mortality cases affected by SARS-CoV2: a systematic review and meta-analysis. Rev. Inst. Med. trop. S. Paulo. 2020; 62(43). 6. Yang J, Zheng Y, Gou X, Pu K, Chen Z, Guo Q, et al. Prevalence of comorbidities and its effects in patients infected with SARS-CoV-2: a systematic review and meta-analysis. Int J Infect Dis. 2020 Maio; 94:91-95.

7. COHEN, Ricardo. Obesidade, diabetes e cia: as pandemias que pioram a COVID-19. Jornal da Sociedade Brasileira de Cirurgia Bariátrica e Metabólica. Maio 2020:1-6.

8. Tyrrell DA, Bynoe ML, Hoorn B. Cultivation of "difficult" viruses from patients with common colds. Lancet. 1966 Janeiro;

1:76-77.

9. Mousavizadeh L, Ghasemi S. Genotype and phenotype of COVID-19: Their roles in pathogenesis. Journal of Microbiology, Immunology and Infection. 2020 Março;:1-5.

10. Tortorici MA, Veesler D. Structural insights into coronavirus entry. Advances in virus research. 2019; 105:93-116.

11. Guo YR, Cao QD, Hong ZS, Tan YY, Chen SD, Jin HJ, et al. The origin, transmission and clinical therapies on coronavirus disease 2019 (COVID-19) outbreak- An update on the status. Military Medical Research. 2020 Março; 7:1-10.

12. Schoeman D, Fielding BC. Coronavirus envelope protein: current knowledge. Virol J. 2019 Maio; 16(69):1-30.

13. Morganna E, Ferreira S, Souza B, Wallace P, Silva P, Miranda W, et al. SARS-CoV-2 aspects related to biology, propagation and transmission of the emerging disease COVID-19. Revista desafios. 2020 Abril; 7:1-9. 
14. Lima LNGC, Sousa MS.; Lima KVB. As descobertas genômicas do SARS-CoV-2 e suas implicações na pandemia de COVID19. Journal of Health \&Biological Sciences. 2020 May; 8(1). doi: 10.12662/2317-3076jhbs.v8i1.3232.p1-9.2020

15. South AM, Diz DI, Chappell MC. COVID-19, ACE2, and the cardiovascular consequences. Am J Physiol Heart Circ Physiol. 2020;318(5):H1084-H1090. doi:10.1152/ajpheart.00217.2020

16. HAYDENMR. Endothelial activation and dysfunction in metabolic syndrome, type 2 diabetes and coronavirus disease 2019. Journal of International Medical Research. 2020; 48(7).

17. Bloomgarden ZT: Diabetes and COVID-19. J Diabetes. 2020; 12:347-348.

18. Fadini GP, Morieri ML, Longato E, Avogaro A. Prevalence and impact of diabetes among people infected with SARS-CoV2. J Endocrinol Invest. 2020. doi: 10.1007/s40618-020-01236-2

19. Xie J, Tong Z, Guan X, Du B, Qiu H. Clinical characteristics of patients who died of coronavirus disease 2019 in China. JAMA Netw Open. 2020;3(4).

20. Hussain A, Bhowmik B,Moreira MCdV. COVID-19 and diabetes: knowledge in progress Diabetes Res Clin Pract.2020 Apr; 162:108-142. doi: 10.1016/j.diabres.2020.108142

21. Roncon L, Zuin M, Rigarelli G, Zuliani G. Diabetic patients with COVID-19 infection are at higher risk of ICU admission and poor short-term outcome J Clin Virol. 2020; 127:104-354. doi: 10.1016/j.jcv.2020.104354

22. Hill MA, Mantzoros C, Sowers JR. Commentary: COVID-19 in patients with diabetes Metabolism. 2020 Mar; 107:154217. doi: 10.1016/j.metabol.2020.154217

23. American Diabetes Association. How COVID-19 Impacts People with Diabetes.Arlington (VA);2020.

24. Li B,Yang J, Zhao F, Zhi L, Wang X, Liu L. Prevalence and impact of cardiovascular metabolic diseases on COVID-19 in China. Clin Res Cardiol. 2020. doi: 10.1007/s00392-020-01626-9 Epub 2020/03/13 PubMed

25. Vracko R, Thorning D, Huang TW. Basal lamina of alveolar epithelium and capillaries: quantitative changes with aging and in diabetes mellitus. Am Rev Respir Dis. 1979; 120(5):973-983.

26. Ofulue AF, Thurlbeck WM. Experimental diabetes and the lung. II. In vivo connective tissue metabolism. Am Rev Respir Dis. 1988;138(2):284-289.

27. Farina J, Furio V, Fernandez-Acenero MJ, Muzas MA. Nodular fibrosis of the lung in diabetes mellitus. Virchows Arch. 1995;427(1):61-63.

28. Pitocco D, Fuso L, Conte EG. The diabetic lung--a new target organ?. Rev Diabet Stud. 2012;9(1):23-35.

doi:10.1900/RDS.2012.9.23

29. DavisW, Knuiman M, Kendall P, Grange V, Davis T. Glycemic Exposure Is Associated With Reduced Pulmonary Function in Type 2 Diabetes The Fremantle Diabetes Study. Diabetes care. 2004;27(3): 752-757. doi:10.2337/diacare.27.3.752.

30. Delamaire M, Maugendre D, Moreno M, Le Goff M-C, Allannic H, Genetet B. Impaired leucocyte functions in diabetic patients. Diabet Med. 1997;14(1):29-34.

31. Summers KL, Marleau AM, Mahon JL, McManus R, Hramiak I, Singh B. Reduced IFN- $\alpha$ secretion by blood dendritic cells in human diabetes. Clin Immunol. 2006;121(1):81-89.

32. Unnikrishnan R, Saboo B, Kesavadev J, Deshpande N, Aravind SR, Joshi S, Anjana RM, Hussain A, Mohan V. Diabetes and coronavirus disease-2019 (COVID-19). J Diabetol. 2020;11:52-6.

33. Hodgson K, Morris J, Bridson T, Govan B, Rush C, Ketheesan N. Immunological mechanisms contributing to the double burden of diabetes and intracellular bacterial infections. Immunology. 2015;144(2):171-185.

34. Saler T, Cakmak G, Saglam ZA, et al. The assessment of pulmonary diffusing capacity in diabetes mellitus with regard to microalbuminuria. Intern Med. 2009; 48: 1939-1943.

35. Drucker DJ. Coronavirus Infections and Type 2 Diabetes-Shared Pathways with Therapeutic Implications. Endocrine Reviews. 2020 June;41(3):457-470.doi:10.1210/endrev/bnaa011

36. Vuille-dit-Bille RN, Camargo SM, Emmenegger L, et al. Human intestine luminal ACE2 and amino acid transporter expression increased by ACE-inhibitors. Amino Acids. 2015;47(4):693-705.

37. Knapp S. Diabetes and infection: is there a link? - A mini-review. Gerontology. 2013;59 (2):99-104. doi:

10.1159/000345107

38. Petrie JR, Guzik TJ, Touyz RM. Diabetes, Hypertension, and Cardiovascular Disease: Clinical Insights and Vascular Mechanisms. Can J Cardiol. 2018;34(5):575-584. doi:10.1016/j.cjca.2017.12.005

39. Brufsky A. Hyperglycemia, hydroxychloroquine, and the COVID-19 epidemic. J Med Virol.2020;92(7):770-775.

doi:10.1002/jmv.25887

40. Rebello CJ, Kirwan JP, Greenway FL. Obesity, the most common comorbidity in SARS-CoV-2: is leptin the link?. Int J Obes.2020;44:1810-1817. doi:10.1038/s41366-020-0640-5

41. Wang Z, Du Z, Zhu F. Glycosylated hemoglobin is associated with systemic inflammation, hypercoagulability, and prognosis of COVID-19 patients. Diabetes Res Clin Pract. 2020;164. doi:10.1016/j.diabres.2020.108214

42. Singh AK, Khunti K. Assessment of risk, severity, mortality, glycemic control and antidiabetic agents in patients with diabetes and COVID-19: A narrative review. Diabetes Res. Clin. Pract. 2020;165. doi:10.1016/j.diabres.2020.108266 43. Souza TA, Siqueira BS, Grassiolli S. Obesidade, comorbidades e COVID19: uma breve revisão de literatura. Rev. Var. 
Scien.: Ciências da Saúde. 2020;6(1):5-8.

44. Ryan DH, Ravussin E, Heymsfield S. COVID 19 and the Patient with Obesity: The Editors Speak Out. Wil. Pub. Heal. Emerg. Collec: Public Health Emergency COVID-19 Initiative. 2020 Apr 01;00(00):1. doi:10.1002/oby.22808

45. Ministério da Saúde. Programa de Evidências para Políticas e Tecnologias de Saúde (PEPTS) - Fundação Oswaldo Cruz (FIOCRUZ). Nota rápida de evidência: observações sobre condições de risco para o agravamento ou morte por COVID-19. Brasília (DF), 2020.

46. Rezende LFM, Thome B, Schveitzer MC, Souza-Júnior PRB de, Szwarcwald CL. Adults at high-risk of severe coronavirus disease-2019 (Covid-19) in Brazil. Rev Saude Publica. 2020;54:50. doi:10.11606/s1518- 8787.2020054002596 47. Louisiana Department of Health. Louisiana Coronavirus COVID-19. Louisiana Department of Health Updates for 3/31/2020. Baton Rouge, (LA); 2020.

48. Centers for Disease Control and Prevention. Coronavirus Disease 2019 (COVID-19). Groups at higher risk for severe illness. Georgia (USA); 2020.

49. Ryan PMD, Caplice NM. Is Adipose Tissue a Reservoir for Viral Spread, Immune Activation, and Cytokine Amplification in Coronavirus Disease 2019?. Cent. for Res. in Vasc. Biol. 2020 May 31;28(7):1. doi:10.1002/oby.22843

50. Francisqueti FV, Nascimento AF, Corrêa CR. Obesidade, inflamação e complicações metabólicas. Nutrire. 2015 Abril;40(1):2-5.

51. Zabetakis I, Lordan R, Norton C, Tsoupras A. Covid-19: The inflammation link and the role of nutrition in potential mitigation. Nutrients 2020; 12:1-28.

52. Prado WL, Lofrano MC, Oyama LM, Dâmaso AR. Obesidade e Adipocinas Inflamatórias: Implicações Práticas para a Prescrição de Exercício. Rev Bras Med Esporte. 2009 set/out;15(5):3.

53. Heialy SA, Hachim M, Senok A, Tayoun AA, Hamoudi R, Alsheikh-Ali A, Hamid Q. Regulation of angiotensin converting enzyme 2 (ACE2) in obesity: implications for COVID-19. BioRxiv: The Preprint server for Biology. 2020 Apr 18;4(17):3-4. doi:10.1101/2020.04.17.046938

54. De Lorenzo A, Maiolo C, Mohamed El, Andreoli A, Petrone De Luca P, Rossi P. Body composition analysis and changes in airways function in obese adults after hypocaloric diet. Chest. 2001;119(5):1409-1415.

55. Zerah F, Harf A, Perlemuter L, Lorino H, Lorino AM, Atlan G. Effects of obesity on respiratory resistance. Chest 1993;103:1470-1476

56. Slutzky LC. Fisioterapia respiratória nas enfermidades neuromusculares. Rio de Janeiro: Revinter; 1997

57. Magnani K, Souza CE, Amorim GC, Santos SB. Função respiratória em obesos e obesos mórbidos. Rev. Uningá. 2004 jul./dez.;(2):2.

58. Sun J, Aghemo A, Forner A, Valenti L. COVID-19 and liver disease. Liver Int. 2020;40(6):1278-1281.

doi:10.1111/liv.14470

59. Fix OF, et al. American Association for the Study of Liver Diseases. Clinical insights for hepatology and liver transplant providers during COVID-19 pandemic. Mar 2020

60. Bomfim JHGG, Gonçalves JS. Suplementos alimentares, imunidade e COVID-19: qual a evidência?. Vittalle - Rev de Cienc da Saúde. 2020;32(1):10-21.doi:10.14295/vittalle.v32i1.11282

61. Berthon BS, Wood LG. Nutrition and Respiratory Health - Feature Review. Nutrients. 2015; 7: 1618- 1643.

62. Alecrim JVC. Políticas públicas de esporte e lazer na promoção de saúde e COVID-19: o que devemos aprender para o futuro. Boletim de Conjuntura (BOCA). 2020;2(5). doi:10.5281/zenodo.3774408

63. Guthold R, Stevens GA, Riley LM, Bull FC. Worldwide trends in insufficient physical activity from 2001 to 2016: a pooled analysis of 358 population-based surveys with 1.9 million participants. Lancet Global Health. 2018;6(10).

64. World health Organization. WHO Report on the Global Tobacco Epidemic, 2008: the MPOWER package. Genova: WHO; 2008.

65. Pinto M, Bardach A, Palacios A,Biz A, Alcaraz A, Rodriguez B, Augustovski F, Pichon-Riviere A. Carga do tabagismo no Brasil e benefício potencial do aumento de impostos sobre os cigarros para a economia e para a redução de mortes e adoecimento. Cad Saúde Pública. 2019;35(8). doi:10.1590/0102-311x00129118

66. Szklo AS, Bertoni N. Relação entre a Epidemia de Tabagismo e a Epidemia recente de Covid-19: um Panorama Atual das Evidências Científicas. Revista Brasileira de Cancerologia. 2020;66. doi: 10.32635/2176-

9745.RBC.2020v66nTemaAtual.1105.

67. Chagas C, Paula TCS, Martins LB. O aumento do consumo de álcool em tempos de pandemia: mídia e normas sociais. Com. Ciências Saúde 2020;31(1):116-120.

68. Rehm J, Kilian C, Ferreira-Borges C, Jernigan D, Monteiro M, Parry CDH, Sanchez ZM, Manthey J. Alcohol use in times of the COVID 19: Implications for monitoring and policy. Drug Alcohol Rev. 2020;39(4):301-304.

69. Nogueira CJ, Cortez ACL, Leal SMO, Dantas EHM. Precauções e recomendações para a prática de exercício físico em face do COVID-19: uma revisão integrativa. 2020.

70. Tavares FE, Santos SMV. O exercício físico e a Covid-19: Quando o Trabalho conduz ao Sedentarismo e substitui a Atividade Física. Id Online - Revista Multidisciplinar e de Psicologia. jul 2020. 
71. Silva ALO, Moreira JC, Martins SR. COVID-19 e tabagismo: uma relação de risco. Cad de Saúde Pública. 2020;36(5). 72. 60- Smith JC, Sausville EL, Girish V, Yuan ML, Vasudevan A, John KM, Sheltzer JM. Cigarette smoke exposure and inflammatory signaling increase the 14xpression of the SARS-CoV-2 receptor ACE2 in the respiratory tract. Developmental Cell. 2020;53(5):514-529. doi: 10.1016/j.devcel.2020.05.012

73. Costa REAR, Paulo AS, Vieira AL, Balsamo ACM, Brito ACS, Mesquita MM. Principais correlações do tabagismo com a COVID-19. Research, Society and Development. 2020;9(9).

74. Lau JT, Yang X, Pang E, Tsui HY, Wong E, Wing YK. SARS-related perceptions in Hong Kong. Emerg Infect Dis. 2005;11:417424.

75. Montagnani JM, Menezes CRS, Pinge-Filho P. Abordagem do alcoolismo e do sistema imunológico nos livros didáticos de ciência e fatores associados ao consumo de álcool por estudantes do Colégio Estadual "Barão do Rio Branco", Londrina, Paraná. Programa de Desenvolvimento Educacional. 2009.

76. Andrade MC, Martins-Filho A, Coelho-Neto J, Mesquita ON, Faria AMC. The long-term impaired macrophages functions are already observed early after high dose ethanol administration. Scandinavian Journal of Immunology. 2008;68:306-314. 77. Goral J, Karavitis J, Kovacs EJ. Exposure-dependent effects of ethanol on the innate immune system. Alcohol. 2008;42:237-247.

78. Souza EG, França EL, Honório-França AC. Efeitos deletérios do etanol sobre o sistema imune: uma breve revisão. Revista Panorâmica On-Line, Barra do Garças - MT; dez 2015;19:56-69. 